\title{
COMMUNICATIONS
}

\section{Orbital free $a b$ initio molecular dynamics study of liquid Al near melting}

\author{
D. J. González, ${ }^{\text {a) }}$ L. E. González, and J. M. López \\ Departamento de Física Teórica, Universidad de Valladolid, Valladolid, Spain \\ Malcolm J. Stott \\ Department of Physics, Queen's University, Kingston, Ontario K7L 3N6, Canada
}

(Received 16 January 2001; accepted 14 July 2001)

The orbital free ab initio molecular dynamics method is applied to study the static and dynamic structure of liquid $\mathrm{Al}$ near the triple point. The method uses a new kinetic energy functional, along with a local pseudopotential constructed within the same kinetic energy functional. The results obtained for the dynamic structure factor are compared with recent experimental data. (C) 2001 American Institute of Physics. [DOI: 10.1063/1.1389473]

\section{INTRODUCTION}

Molecular dynamics (MD) has become a useful technique for studying the properties of liquid systems. Whereas classical molecular dynamics (CMD) techniques require inter-particle potentials in which the electronic degrees of freedom are hidden, the ab initio molecular dynamics (AIMD) methods compute the forces acting on the nuclei from electronic structure calculations which are performed as the MD trajectory is generated. Lately, there has been a large increase in the application of AIMD methods based on the density functional theory (DFT), ${ }^{1,2}$ which allows, for given nuclear positions, the calculation of the ground state electronic energy of a collection of atoms, and also yields the forces on the nuclei via the Hellmann-Feynman (HF) theorem. Consequently, MD simulations can be performed in which the nuclear positions evolve according to classical mechanics while the electronic subsystem follows adiabatically.

We present results of an AIMD simulation on the static and dynamic properties of liquid $\mathrm{Al}$ near the triple point. Most studies on this system have been performed by CMD simulations combined with a variety of interatomic pair potentials. ${ }^{3-5}$ The results obtained showed fair agreement with the experimental data, with some discrepancies appearing for either the static structure factor ${ }^{3,4}$ or the dynamic structure factor. ${ }^{5}$ A recent AIMD simulation ${ }^{6}$ focusing on the liquid static structural properties only, obtained results in good agreement with experiment. Here, we test the ability of the method to describe the dynamical properties.

\section{THEORY}

The total potential energy of $N$ ions with valence $Z$, enclosed in a volume $V$, and interacting with $N_{e}=N Z$ valence electrons through a local electron-ion potential $v(r)$, is written, within the Born-Oppenheimer approximation, as the

\footnotetext{
${ }^{a)}$ Electronic mail: david@liq1.fam.cie.uva.es
}

sum of the direct ion-ion Coulombic interaction energy and the ground state energy of the electronic system subject to the external potential created by the ions, $V_{\text {ext }}\left(\mathbf{r},\left\{\mathbf{R}_{l}\right\}\right)$ $=\sum_{i=1}^{N} v\left(\left|\mathbf{r}-\mathbf{R}_{i}\right|\right)$,

$$
E\left(\left\{\mathbf{R}_{l}\right\}\right)=\sum_{i<j} \frac{Z^{2}}{\left|\mathbf{R}_{i}-\mathbf{R}_{j}\right|}+E_{g}\left[\rho_{g}(\mathbf{r}), V_{\mathrm{ext}}\left(\mathbf{r},\left\{\mathbf{R}_{l}\right\}\right)\right],
$$

where $\rho_{g}(r)$ is the ground state electronic density and $\mathbf{R}_{l}$ are the ionic positions. According to DFT, the ground state electronic density minimizes the energy functional

$$
E[\rho(\mathbf{r})]=T_{s}[\rho]+E_{\mathrm{ext}}[\rho]+E_{H}[\rho]+E_{\mathrm{xc}}[\rho],
$$

where the terms represent the electronic kinetic energy, $T_{s}[\rho]$, of a noninteracting system with density $\rho(\mathbf{r})$, the electron-ion interaction energy, $E_{\text {ext }}[\rho]$, the classical electrostatic Hartree energy, $E_{H}[\rho]$, and the exchangecorrelation energy, $E_{\mathrm{xc}}[\rho]$, for which we will adopt the local density approximation. In the Kohn-Sham approach to density functional theory (KS-DFT), ${ }^{2,7,8}$ the noninteracting electron kinetic energy, $T_{s}[\rho]$, is calculated exactly using single particle orbitals which yield the electron density $\rho$, but at the cost of a huge computational effort. However, this can be alleviated somewhat by adopting an orbital-free approach in which an explicit but approximate density functional for $T_{s}[\rho]$ is used. ${ }^{1,9}$ Although the von Weizsäcker term, $T_{W}[\rho(\mathbf{r})]=\frac{1}{8} \int d \mathbf{r}|\nabla \rho(\mathbf{r})|^{2} / \rho(\mathbf{r})$, is thought to be essential for a good description of the kinetic energy in the limit of rapidly varying density, ${ }^{10}$ other terms are usually incorporated so as to reproduce correctly some exactly known limits. In the uniform density limit, the kinetic energy is given by the Thomas-Fermi functional, $T_{\mathrm{TF}}[\rho(\mathbf{r})]=\frac{3}{10} \int d \mathbf{r} \rho(\mathbf{r})$ $\times k_{F}(\mathbf{r})^{2}$, where $k_{F}(\mathbf{r})=\left(3 \pi^{2}\right)^{1 / 3} \rho(\mathbf{r})^{1 / 3}$ is the local Fermi wave vector; and for an almost uniform density, linear response theory (LRT) becomes correct, with a response function given by the Lindhard function.

The computational advantages of the orbital-free $a b i n i-$ tio molecular dynamics (OF-AIMD) simulations have stimu- 
lated the development of accurate kinetic energy functionals. ${ }^{9}$ We mention particularly the form proposed by Garcí a-González et al. ${ }^{11}$ which we have used in this work in the following simplified version: $T_{s}=T_{W}+T_{\beta}$,

$$
\begin{aligned}
& T_{\beta}=\frac{3}{10} \int d \mathbf{r} \rho(\mathbf{r})^{5 / 3-2 \beta} \widetilde{k}(\mathbf{r})^{2} \\
& \tilde{k}(\mathbf{r})=\left(2 k_{F}^{0}\right)^{3} \int d \mathbf{s} k(\mathbf{s}) w_{\beta}\left(2 k_{F}^{0}|\mathbf{r}-\mathbf{s}|\right),
\end{aligned}
$$

where $k(\mathbf{r})=\left(3 \pi^{2}\right)^{1 / 3} \rho(\mathbf{r})^{\beta}, k_{F}^{0}$ is the Fermi wave vector corresponding to a mean electron density $\rho_{0}$, and $w_{\beta}(x)$ is a weight function determined by requiring the correct recovery of both the LRT limit and the uniform density limit. It is most important to note that this functional is positive definite for any value of $\beta$. Moreover, in the $k_{F}^{0} \rightarrow 0$ limit when the mean electron density vanishes (for instance in a finite system), the von Weizsäcker term is recovered if $\beta=4 / 9$, whereas for other values of $\beta$, the limit is $T_{W}+C T_{\mathrm{TF}}$. In these simulations we have used a value of $\beta=0.51$, close to $4 / 9$ and for which the coefficient $C$ in the $k_{F}^{0} \rightarrow 0$ limit is 0.046. This guarantees that minimization of the energy can be achieved for the thermodynamic states we consider.

Another key ingredient of the energy functional is the ionic pseudopotential, $v_{\mathrm{ps}}(r)$, used to describe the ionelectron interaction. We sketch the main steps in its construction and further details will be given elsewhere. ${ }^{12}$ First, the electronic density distribution is constructed for a model system composed of an homogeneous electron gas (jellium) of density $\rho_{0}=N_{e} / V$, in which a spherical cavity has been made and where an Al nucleus has been inserted. This is performed using the KS-DFT approach to obtain the core and valence electronic states. The latter ones are used to determine the change, $n(r)$, in the valence electron density induced in the jellium by the presence of the $\mathrm{Al}$ nucleus and the cavity - the displaced valence electron density. Now, $r^{2} n(r)$ exhibits rapid oscillations for small $r$, because of the orthogonality constraint between valence and core electronic wave functions with the same angular momentum. But the $n(r)$ will be used to construct a local pseudopotential which, by its very definition, makes impossible the appearance of orthogonality oscillations as no bound states can exist; therefore, they are removed as shown in Ref. 13. Next, an effective local pseudopotential, $v_{\mathrm{ps}}(r)$, is constructed which when inserted into the jellium together with the cavity, reproduces, within the OF-DFT procedure, the same displaced valence electronic density. This is achieved by inserting $n(r)$ into the Euler equation, obtained by minimizing the energy functional of Eq. (2), together with the normalization constraint $\int_{V} d \mathbf{r} n(r)=Z$,

$$
\frac{\delta T_{s}[\rho]}{\delta \rho(\mathbf{r})}+\frac{\delta E_{\mathrm{ext}}[\rho]}{\delta \rho(\mathbf{r})}+\frac{\delta E_{H}[\rho]}{\delta \rho(\mathbf{r})}+\frac{\delta E_{x c}[\rho]}{\delta \rho(\mathbf{r})}-\mu=0,
$$

where $\rho(r)=\rho_{0}+n(r)$ and $\mu$ is a Lagrange multiplier chosen so that the pseudopotential vanishes at large distances. Moreover, due to the spherical symmetry of the system, all the magnitudes depend on $|\mathbf{r}| \equiv r$.

In our simulations we consider $N$ ions in a cubic cell with periodic boundary conditions. Given the positions of the

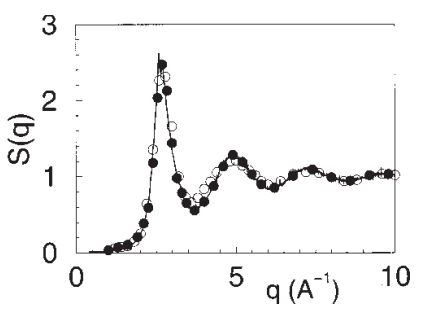

FIG. 1. Static structure factors of liquid $\mathrm{Al}$ at $943 \mathrm{~K}$. Full circles: experimental x-ray diffraction data (14 and 15). Open circles: experimental neutron diffraction data (16). Continuous line: OF-AIMD simulations.

ions at time $t$, the electronic density is expanded in plane waves, the energy functional is minimized with respect to the Fourier coefficients of the expansion, yielding the ground state electronic density and energy, and finally the HF theorem is used to obtain the forces on the ions which along with the Verlet leapfrog algorithm are used to update the ionic positions and velocities.

\section{RESULTS AND DISCUSSION}

Liquid Al has been simulated at a thermodynamic state close to the triple point for which $T=943 \mathrm{~K}$ and the number density, $\rho=0.0529 \AA^{-3}$. We have used 205 particles, the time step was $0.001 \mathrm{ps}$, the equilibration lasted for $10 \mathrm{ps}$, and the calculation of properties was made averaging over another $30 \mathrm{ps}$. The cutoff energy for the plane-wave expansion was $30 \mathrm{Ry}$.

The obtained static structure factor, $S(q)$, is shown in Fig. 1, along with the corresponding experimental data measured by $\mathrm{x}$-ray ${ }^{14,15}$ and neutron diffraction. ${ }^{16}$ Comparison shows small differences in the region $2 \AA^{-1} \leqslant q \leqslant 5 \AA^{-1}$, with the neutron values being slightly greater than the $x$-ray ones and with our OF-AIMD results sitting remarkably well between both sets of experimental data, although closer to the x-ray results. Moreover, by the relation $S(q \rightarrow 0)$ $=\rho k_{B} T \kappa_{T}$, we have determined the isothermal compressibility, $\kappa_{T}$, obtaining a value of 2.37 (in $10^{-11} \mathrm{~m}^{2} \mathrm{~N}^{-1}$ units) which is close to the experimental value ${ }^{17}$ of 2.43 . Figure 2 shows the results obtained for the normalized velocity autocorrelation function of a tagged ion in the fluid, $Z(t)=\left\langle\mathbf{v}_{1}(t) \mathbf{v}_{1}(0)\right\rangle /\left\langle v_{1}^{2}\right\rangle$. It exhibits the typical backscattering behavior, along with other features similar to those of the simple metals near melting, ${ }^{18,19}$ namely, the deepness of the first minimum is around 0.20 and the following maximum is rather weak, with a maximum value very close to zero; in fact, a very similar shape, although with a narrower first

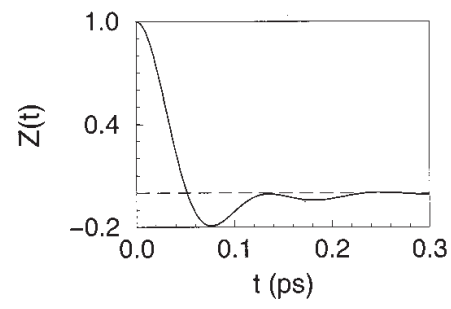

FIG. 2. OF-AIMD result for the normalized velocity autocorrelation function of liquid $\mathrm{Al}$ at $943 \mathrm{~K}$. 


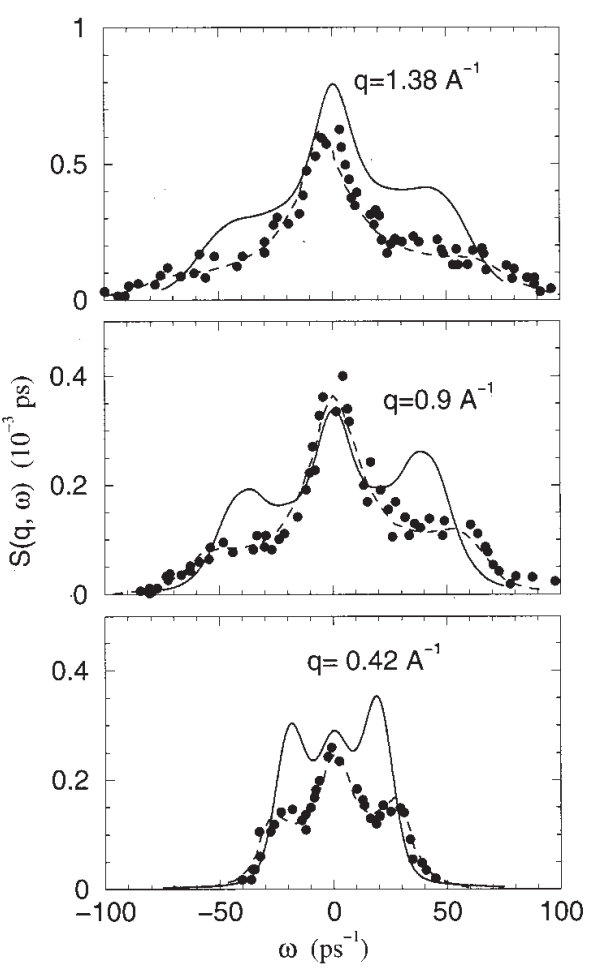

FIG. 3. Dynamic structure factor, $S(q, \omega)$, for several $q$ values, for liquid aluminum at $T=943 \mathrm{~K}$. Continuous line: OF-AIMD simulations. Full circles: experimental data (Ref. 22). Dashed line: best fit to the experimental data.

minimum, was also obtained in the CMD simulations. ${ }^{5}$ The self-diffusion coefficient, $D$, can be found by either the time integration of $Z(t)$ or from the slope of the mean square displacement $\delta R^{2}(t) \equiv\left\langle\left|\mathbf{R}_{1}(t)-\mathbf{R}_{1}(0)\right|^{2}\right\rangle$ of a tagged ion in the fluid. These lead, respectively, to $D_{\mathrm{OF}-\mathrm{AIMD}}=0.53$ and $0.56 \AA^{2} / \mathrm{ps}$. Unfortunately, no experimental data are available for the $D$ of liquid $\mathrm{Al}$ and we can only compare with the results of a full KS-DFT calculation ${ }^{20}$ for liquid Al near the triple point, using 64 particles and a non-local BacheletHamann-Schluter pseudopotential, ${ }^{21}$ which gave $D_{\text {KS-DFT }}$ $=0.60 \AA^{2} / \mathrm{ps}$, derived from the slope of the corresponding $\delta R^{2}(t)$. We note that the CMD simulations ${ }^{5}$ with several pair potentials gave values within the range $0.41-0.45 \AA^{2} / \mathrm{ps}$.

A key role in the dynamical properties is played by the dynamic structure factor $S(q, \omega)$, which embodies the collective dynamics of density fluctuations. It has been obtained by a time Fourier transform of the intermediate scattering function, $F(q, t)$, which is the quantity directly evaluated in the OF-AIMD simulations. The calculated $S(q, \omega)$, convoluted with the experimental resolution function and including the detailed balance condition are compared in Fig. 3 with the experimental inelastic x-ray scattering (IXS) data of Scopigno et al., ${ }^{22}$ for liquid $\mathrm{Al}$ at $T=1000 \mathrm{~K}$. Although the agreement is generally good, there are discrepancies in the height of the central and side peaks, but the positions of the side peaks are fairly well reproduced. Indeed the calculated $S(q, \omega)$ show well defined side peaks (representative of the existence of collective density excitations) up to $q \approx 1.6$ $\AA^{-1}$, which amounts to $\approx 3 / 5 q_{p}$. Similar results, although

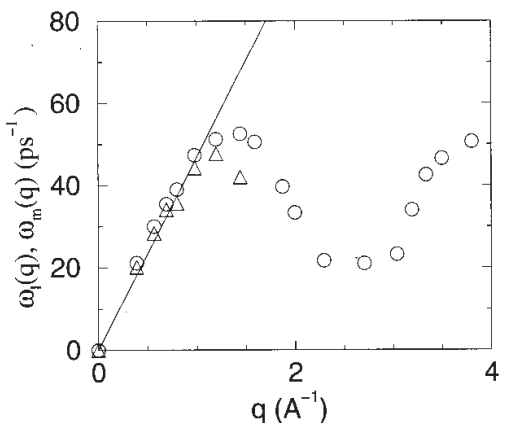

FIG. 4. Dispersion relation for liquid $\mathrm{Al}$ at $T=943 \mathrm{~K}$. Open triangles: peak positions, $\omega_{m}(q)$, from the OF-AIMD $S(q, \omega)$. Open circles: peak positions, $\omega_{l}(q)$, from the maxima of the OF-AIMD longitudinal current, $J_{l}(q, \omega)$. Full line: linear dispersion with the hydrodynamic adiabatic sound velocity, $v=4700 \mathrm{~m} / \mathrm{s}$ (Ref. 17).

with a better description of the central peak at the lowest $q$-values, were also obtained in the CMD simulations; ${ }^{5}$ nevertheless, it must be noted that the $\operatorname{CMD} F(q, t)$ were previously fitted to an analytical expression interpolating between the ideal gas and viscoelastic models, and therefrom the $S(q, \omega)$ were derived. From the positions of the side peaks, $\omega_{m}(q)$, the dispersion relation of the density fluctuations has been obtained and is shown in Fig. 4, along with the dispersion relation obtained from the maxima, $\omega_{l}(q)$, of the current correlation function, $J_{l}(q, \omega)=\omega^{2} S(q, \omega)$.

In the small- $q$ region, the slope of the dispersion relation gives the adiabatic sound velocity, $c_{s}(q)=v_{\text {th }} \sqrt{\gamma / S(q)}$, where $v_{\text {th }}$ is the thermal velocity and $\gamma$ is the ratio of the specific heats. In the limit $q \rightarrow 0$, this quantity reduces to the bulk adiabatic sound velocity. Extrapolating the OF-AIMD results for $S(q)$ and using the experimental value ${ }^{23}$ of $\gamma$ $\approx 1.25$, we obtain a value of $\approx 4850 \mathrm{~m} / \mathrm{s}$ for the bulk adiabatic sound velocity which compares reasonably well with the experimental value ${ }^{17}$ of $\approx 4700 \mathrm{~m} / \mathrm{s}$. Figure 4 shows a positive dispersion, i.e., an increase of $\omega_{l}(q)$ with respect to the values predicted by the hydrodynamic adiabatic sound velocity. Similar behavior is exhibited in the IXS data of Scopigno et al. ${ }^{22}$ with a maximum at $\approx 0.45 \AA^{-1}$. Moreover, this effect has also been observed experimentally in other liquid metals, i.e., $\mathrm{Li}, \mathrm{Na}, \mathrm{Rb}$, and $\mathrm{Cs}^{24}$

We conclude by signaling that the above-given results suggest that liquid Al exhibits dynamical features rather similar to those already observed in the liquid alkali metals. Moreover, the good agreement with the available experimental results supports the suitability of the OF-AIMD method to describe the dynamical features of liquid $\mathrm{Al}$ near melting. The discrepancies found for the dynamic structure factor may indicate, in our opinion, the need for improvements in the ionic pseudoptential. Further work in that direction is in progress.

\section{ACKNOWLEDGMENTS}

Work supported by the Junta de Castilla y León (VA70/ 99), CICYT (PB98-0368-CO2), NATO (CRG971173) and the British-Spanish Joint Research Program (No. HB1997- 
0188). D.J.G. acknowledges the University of Valladolid for financial support. M.J.S. acknowledges the support of the NSERC of Canada.

${ }^{1}$ P. Hohenberg and W. Kohn, Phys. Rev. 136, 864 (1964).

${ }^{2}$ W. Kohn and L. J. Sham, Phys. Rev. 140, A1133 (1965).

${ }^{3}$ G. Jacucci, R. Taylor, A. Tenenbaum, and N. van Doan, J. Phys. F: Met. Phys. 11, 783 (1981).

${ }^{4}$ J. Hafner and W. Jank, Phys. Rev. A 42, 11530 (1990).

${ }^{5}$ I. Ebbsjo, T. Kinell, and J. Waller, J. Phys. C 13, 1865 (1980).

${ }^{6}$ J. A. Anta, B. J. Jesson, and P. A. Madden, Phys. Rev. B 58, 6124 (1998).

${ }^{7}$ R. Carr and M. Parrinello, Phys. Rev. B 55, 2471 (1985).

${ }^{8}$ F. Shimojo, Y. Zempo, K. Hoshino, and M. Watabe, J. Non-Cryst. Solids 205-207, 983 (1996); B. J. Costa Cabral and J. L. Martins, Phys. Rev. B 51, 982 (1995); G. Kresse and J. Hafner, ibid. 55, 7539 (1997).

${ }^{9}$ F. Perrot, J. Phys.: Condens. Matter 6, 431 (1994); E. Smargiassi and P. A. Madden, Phys. Rev. B 49, 5220 (1994); M. Foley and P. A. Madden, ibid. 53, 10589 (1996).

${ }^{10}$ E. S. Kryachko and E. V. Ludeña, Energy Density Functional Theory of Many-Electron Systems (Kluwer Academic, London, 1990), and references therein.

${ }^{11}$ P. Garcia-González, J. E. Alvarellos, and E. Chacćon, Phys. Rev. B 53, 9509 (1996); 54, 1897 (1996); 57, 4857 (1998).

${ }^{12}$ D. J. González, L. E. González, J. M. López and M. J. Stott (unpublished).
${ }^{13}$ L. E. González, D. J. González, M. Silbert, and J. A. Alonso, J. Phys.: Condens. Matter 5, 4283 (1993).

${ }^{14}$ IAMP database of SCM-LIQ, Tohoku University. URL: http:// www.iamp.tohoku.ac.jp/database/scm/LIQ/sq.html

${ }^{15}$ Y. Waseda, The Structure of Non-Crystalline Materials (McGraw-Hill, New York, 1980).

${ }^{16}$ S. Takeda, S. Harada, S. Tamaki, and Y. Waseda, J. Phys. Soc. Jpn. 60, 2241 (1991).

${ }^{17}$ H. J. Seemann and F. K. Klein, Z. Angew. Math. Phys. 19, 368 (1965).

${ }^{18} \mathrm{U}$. Balucani and M. Zoppi, Dynamics of the Liquid State (Clarendon, Oxford, 1994).

${ }^{19}$ M. M. G. Alemany, J. Casas, C. Rey, L. E. González, and L. J. Gallego, Phys. Rev. E 56, 6818 (1997).

${ }^{20}$ P. E. Blochl and M. Parrinello, Phys. Rev. B 45, 9413 (1992).

${ }^{21}$ G. B. Bachelet, D. R. Hamann, and M. Schluter, Phys. Rev. B 26, 4199 (1982); D. R. Hamann, ibid. 40, 2980 (1989).

${ }^{22}$ T. Scopigno, U. Balucani, G. Ruocco, and F. Sette, Phys. Rev. E 63, 011210 (2000).

${ }^{23}$ G. M. B. Webber and R. W. B. Stephens, Physical Acoustics, edited by W. P. Mason (Academic, New York, 1968), Vol. IV B.

${ }^{24}$ H. Sinn and E. Burkel, J. Phys.: Condens. Matter 8, 9369 (1996); H. Sinn, F. Sette, U. Bergmann, Ch. Halcoussis, M. Krisch, R. Verbeni, and E. Burkel, Phys. Rev. Lett. 78, 1715 (1997); T. Scopigno, U. Balucani, G. Ruocco, and F. Sette, J. Phys.: Condens. Matter 12, 8009 (2000); C. Pilgrim, S. Hosokawa, H. Saggau, H., Sinn, and E. Burkel, J. Non-Cryst. Solids 250-252, 96 (1999). 